\title{
Proton Spin Lattice Relaxation in Vinylidene Fluoride/Trifluoroethylene Copolymers II. Effects of Vinylidene Fluoride Content upon Spin Relaxation Processes
}

\author{
Fumiaki ISHII and Akira ODAJIMA \\ Department of Applied Physics, Faculty of Engineering, \\ Hokkaido University, Sapporo 060, Japan
}

(Received January 6, 1986)

\begin{abstract}
In the proton spin lattice relaxation time $T_{1}$ measurements on $\mathrm{VDF} / \mathrm{TrFE}$ copolymers with the VDF contents of 52 and $65 \mathrm{~mol} \%$, three kinds of spin lattice relaxation processes were observed below the Curie temperature $T_{\mathrm{c}}$, around $T_{\mathrm{c}}$ and in a certain temperature region above $T_{\mathrm{c}}$. They were compared with those of $\mathrm{P}\left(\mathrm{VDF}_{72} / \mathrm{TrFE}_{28}\right)$. The thermal hysteresis of $T_{1}$ against temperature for each relaxation process became smaller in order of the VDF contents of 72 , 65 , and $52 \mathrm{~mol} \%$.

The $T_{1}$ in the paraelectric phase for each copolymer was analyzed, on the basis of Bloembergen's theory, in terms of the non-exponential correlation function which describes a onedimensional diffusion motion of conformational defects along the chain. The activation energy of the 1-D diffusion motion was about $8.5 \mathrm{kcal} \mathrm{mol}^{-1}$ which did not depend on the VDF content. The $1 / T_{1}$ and the correlation time of the 1-D diffusion motion for each copolymer were converted, respectively, to those of $\mathrm{P}\left(\mathrm{VDF}_{72} / \mathrm{TrFE}_{28}\right)$ with the scaling factors of $\xi^{-2}$ and $\xi^{-4}$, in which the $\xi$ is the ratio of the VDF content $(\mathrm{mol} \%$ ) to $72 \mathrm{~mol} \%$.

KEY WORDS Vinylidene Fluoride / Trifluoroethylene Copolymer / Vinylidene Fluoride Content / Spin Lattice Relaxation Time / Thermal Hysteresis / Paraelectric Phase / 1-D Diffusion Motion / Conformational Defects / Scaling Factor /
\end{abstract}

It is well known that the copolymer of vinylidene fluoride (VDF) and trifluoroethylene (TrFE) exhibits the ferroelectric phase transition, which strongly depends on the VDF content, and that the thermal hysteresis of the transition appears when the VDF content exceeds $55 \mathrm{~mol} \%$. The thermal hysteresis may be associated with a trans-gauche energy difference $\Delta U\left(=U_{\mathrm{TT}}-U_{\mathrm{TG}}\right)$ of polymer chain. It has been elucidated by Tashiro et $a l .{ }^{1}$ from the infrared absorbances of trans and gauche bonds in the paraelectric phase that as the VDF content increases the $\Delta U$ changes its sign from negative to positive, this is, the conformational stability of the chain changes from trans to gauche. Odajima ${ }^{2}$ corroborated by means of a modified Ising Model that, for the positive energy difference of the trans form to the gauche form, the phase transition is of a first order and its thermal hysteresis also becomes larger. In Part I of this series, ${ }^{3}$ three kinds of the spin-lattice relaxation processes have been investigated in the drawn film of $\mathrm{P}\left(\mathrm{VDF}_{72} / \mathrm{TrFE}_{28}\right)$, which were named as $\beta, \alpha_{t}$, and $\alpha_{b}^{\prime}$ in order of increasing temperature of their appearance. The $\beta$ and $\alpha_{\mathrm{t}}$ relaxations are respectively associated with the motion of the chain backbone in partially crystalline regions and the ferroelectric phase transition. The nuclear spin-lattice relaxation time $T_{1}$ for $\alpha_{\mathrm{b}}^{\prime}$ becomes larger with increasing the resonance angular frequency $\omega$, temperature $T$ and the alignment angle $\gamma$ between the magnetic field $H_{0}$ and the drawn direction of 
the film. The formula for $T_{1}$ in an isolated spin pair modulated by a one-dimentional (1-D) diffusion motion of the conformational defects was derived from Bloembergen's theory, ${ }^{4}$ in terms of the non-exponential correlation function obtained by Hunt and Powles. ${ }^{5}$ Existence of the 1-D diffusion motion in the paraelectric phase ${ }^{6}$ was confirmed from the reasonable agreement between theory and the experimental results.

The purpose of this paper is to report further the $T_{1}$ measurements on the drawn films of $\mathrm{P}\left(\mathrm{VDF}_{52} / \mathrm{TrFE}_{48}\right)$ and $\mathrm{P}\left(\mathrm{VDF}_{65} / \mathrm{TrFE}_{35}\right)$, examining how the VDF content affects three kinds of spin lattice relaxation processes, which are $\beta, \alpha_{\mathrm{t}}$, and $\alpha_{\mathrm{b}}^{\prime}$ described in Part I. ${ }^{3}$ The $T_{1}$ 's for the $\alpha_{b}^{\prime}$ relaxation in both copolymers are analyzed, on the basis of Bloembergen's theory, ${ }^{4}$ in terms of the non-exponential correlation function, and compared with the results of $\mathrm{P}\left(\mathrm{VDF}_{72} / \mathrm{TrFE}_{28}\right){ }^{3}$ Then, we will show that the $1 / T_{1}$ and the correlation time of the local field modulated by the 1-D diffusion motion in each copolymer are converted to those in $\mathrm{P}\left(\mathrm{VDF}_{72} / \mathrm{TrFE}_{28}\right)$ by the scaling factors of $\xi^{-2}$ and $\xi^{-4}$, respectively, in which $\xi$ is the ratio of the VDF content $x(x=52$ or $65 \mathrm{~mol} \%$ ) to that of $72 \mathrm{~mol} \%$.

\section{EXPERIMENTAL}

The samples used were the $\mathrm{VDF}_{x} /$ $\mathrm{TrFE}_{100-x}$ copolymers with the VDF contents of $x=52$, and $65 \mathrm{~mol} \%$, which were supplied by the Daikin Kogyo Co., Ltd. The films of these copolymers were prepared by slowly casting from 2-butanone solutions under atmosphere at room temperature and then were drawn $\times 6$ uniaxially. The drawn films of $\mathrm{P}\left(\mathrm{VDF}_{52} / \mathrm{TrFE}_{48}\right)$ and $\mathrm{P}\left(\mathrm{VDF}_{65} /\right.$ $\mathrm{TrFE}_{35}$ ) were annealed at $130^{\circ} \mathrm{C}$ for $20 \mathrm{~h}$ and $9 \mathrm{~h}$ while pressing them between glass plates. Then, their final drawn ratios were 4 for $\mathrm{P}\left(\mathrm{VDF}_{52} / \mathrm{TrFE}_{48}\right)$ and 4.5 for $\mathrm{P}\left(\mathrm{VDF}_{65} /\right.$ $\mathrm{TrFE}_{35}$ ).

The $T_{1}$ 's in both copolymers were measured for $\gamma=0,45$ and $90^{\circ}$ by using a NMR pulse spectrometer, operating at 20 and $90 \mathrm{MHz}$ over a temperature range from -23 and $120^{\circ} \mathrm{C}$. The processes of the temperature rise from -23 to $120^{\circ} \mathrm{C}$ and the fall from 120 to $-23^{\circ} \mathrm{C}$ are taken as the arrow signs of $\uparrow$ and $\downarrow$, respectively.

A pulse NMR spectrometer, procedures of operation and data reduction of $T_{1}$ have been described elsewhere. ${ }^{3}$

\section{RESULTS}

Figures $1 \mathrm{a}, \mathrm{b}$, and $\mathrm{c}$ show the $\ln T_{1}$ as a function of $T$ for $\gamma=0,45$, and $90^{\circ}$ in P$\left(\mathrm{VDF}_{52} / \mathrm{TrFE}_{48}\right)$. The filled (- - ) and unfilled (-O-) circles are the values of $T_{1}$ in the $\uparrow$ - and $\downarrow$ processes, respectively. The $T_{1} v s$. $T$ curves for $\gamma=0,45$, and $90^{\circ}$ minimize at about $68^{\circ} \mathrm{C}$ in the $\uparrow$ - and $\downarrow$-processes and indicate the concave curves over the whole temperatures. The $\mathrm{P}\left(\mathrm{VDF}_{52} / \mathrm{TrFE}_{48}\right)$ does not almost exhibits an thermal hysteresis of the $T_{1}$ in both $\uparrow$ - and $\downarrow$ processes. In each $T_{1}$ vs. $T$ curve in the $\downarrow$ process, a discontinuity is observed evidently in the temperature region between 55 and $60^{\circ} \mathrm{C}$ $(\downarrow)$, comparing with those in the $\uparrow$-process. Figure 2 shows the $\gamma$-dependence of the $\ln T_{1}$ vs. $T^{-1}$ curve in $\mathrm{P}\left(\mathrm{VDF}_{52} / \mathrm{TrFE}_{48}\right)$ in the $\downarrow$ process. For each orientation, a linear relationship can be observed in the temperature range from 95 to $120^{\circ} \mathrm{C}$ in $\downarrow$-process. In the temperature region between 120 and $40^{\circ} \mathrm{C}(\downarrow)$, the value of $T_{1}$ becomes larger in order of $\gamma=0,45$, and $90^{\circ}$. The $\ln T_{1}$ vs. $T^{-1}$ curves for $\gamma=45$ and $90^{\circ}$ intersect each other at $35^{\circ} \mathrm{C}$.

It is considered that three kinds of the spinlattice relaxations take place in the three temperature ranges of 30 to $50^{\circ} \mathrm{C}, 60$ to $95^{\circ} \mathrm{C}$, and 95 to $120^{\circ} \mathrm{C}$ in both $\uparrow$ - and $\downarrow$-processes. These relaxation processes are referred to as $\beta, \alpha_{t}$, and $\alpha_{b}^{\prime}$ in order of increasing temperature in a similar way to $\mathrm{P}\left(\mathrm{VDF}_{72} / \mathrm{TrFE}_{28}\right){ }^{3}$ In Table I, the temperature ranges of the $\beta, \alpha_{t}$, and $\alpha_{b}^{\prime}$ relaxations in the $\uparrow$ - and $\downarrow$-processes are summarized. 

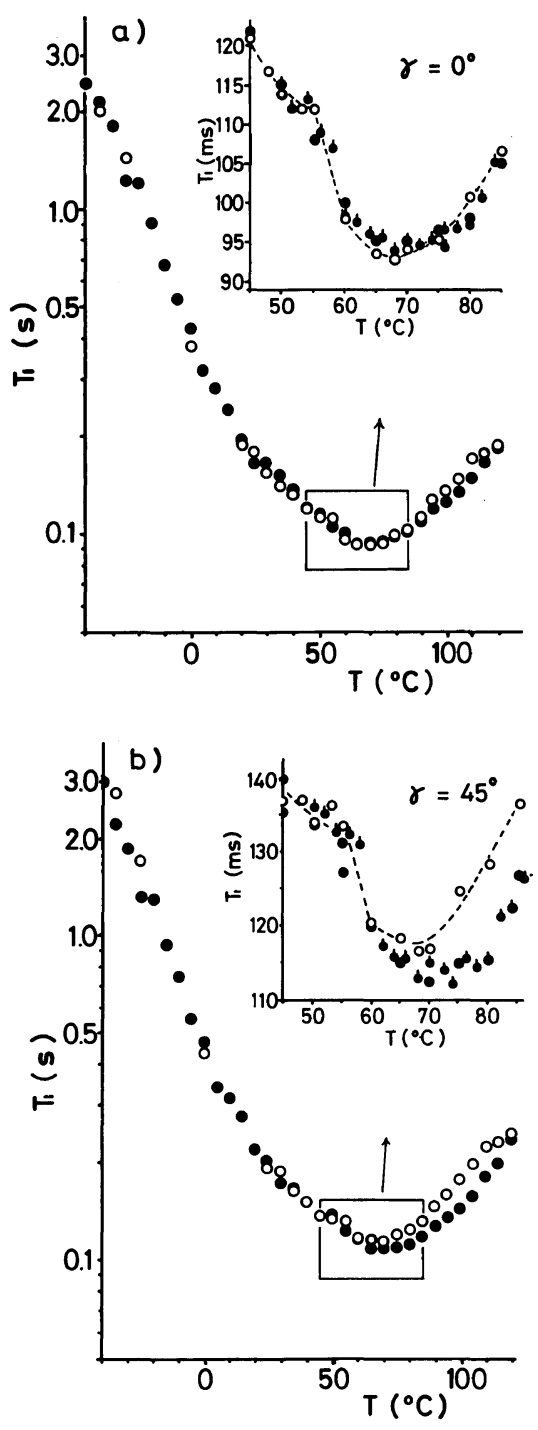

Figures $3 \mathrm{a}, \mathrm{b}$, and $\mathrm{c}$ show the $\ln T_{1}$ as a function of $T$ in $\mathrm{P}\left(\mathrm{VDF}_{65} / \mathrm{TrFE}_{35}\right)$ for $\gamma=0$, 45 , and $90^{\circ}$. In the $\uparrow$-process, they indicate the concave curve over the whole temperatures and minimize at about $89^{\circ} \mathrm{C}(\uparrow)$. The concave curve for each $\gamma$ may be constructed from three kinds of the relaxation processes which are referred to as $\beta(\uparrow), \alpha_{\mathrm{t}}(\uparrow)$, and $\alpha_{\mathrm{b}}^{\prime}(\uparrow)$. No $\beta$ ( $\uparrow$ ) relaxation can be separated from the $\alpha_{\mathrm{t}}$ $(\uparrow)$ relaxation in contrast with that in $\mathrm{P}$ $\left(\mathrm{VDF}_{72} / \mathrm{TrFE}_{28}\right) .{ }^{3}$ Therefore, the temperature region of the relaxation was determined as

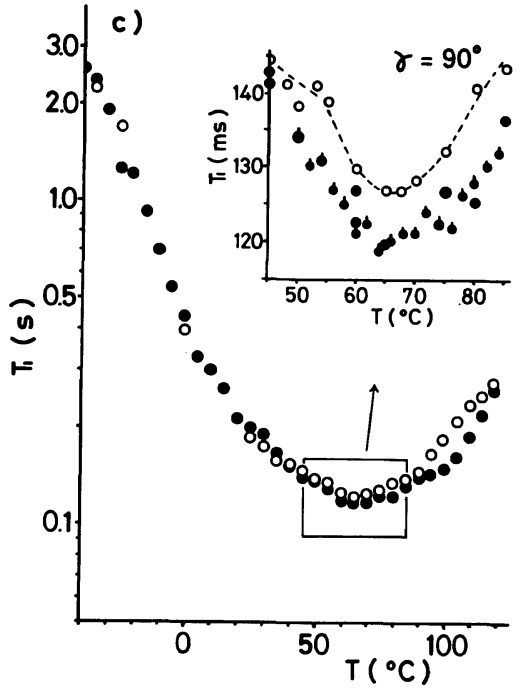

Figure 1. Temperature dependences of $T_{1}$ for $\mathrm{P}\left(\mathrm{VDF}_{52} / \mathrm{TrFE}_{48}\right)$ : (a) $\gamma=0^{\circ}$, (b) $\gamma=45^{\circ}$, and (c) $\gamma=90^{\circ}$. The filled and unfilled circles indicate $T_{1}$ in the $\uparrow$ - and $\downarrow$ processes, respectively. The insets show the behavior of $T_{1}$ between 45 and $95^{\circ} \mathrm{C}$ in the $\uparrow$-process (, , first run;

, second run) and in the $\downarrow$-process (--O--).

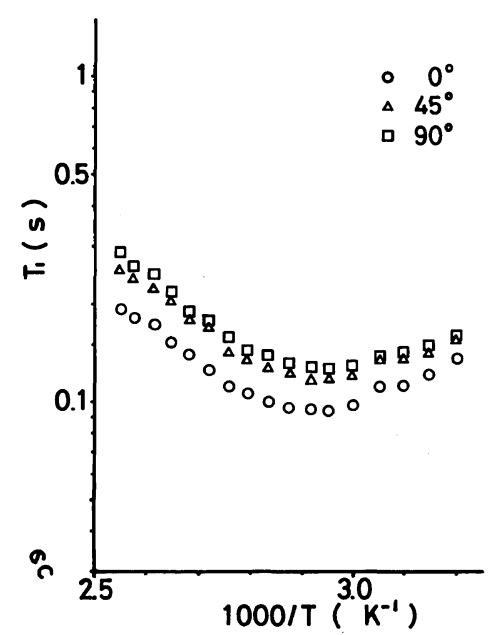

Figure 2. Plots of $T_{1}$ against reciprocal temperature for $\mathrm{P}\left(\mathrm{VDF}_{52} / \mathrm{TrFE}_{48}\right)$ in the cooling process. The filled circles and the unfilled triangles and circles indicate $T_{1}$ for $\gamma=0,45$, and $90^{\circ}$, respectively.

below $79^{\circ} \mathrm{C}$ where a discontinuous line narrowing of the broad width was observed in the line width ${ }^{8}$ measurements. In the $\downarrow$-process, the $T_{1}$ value for each angle $\gamma$ is lower than that 

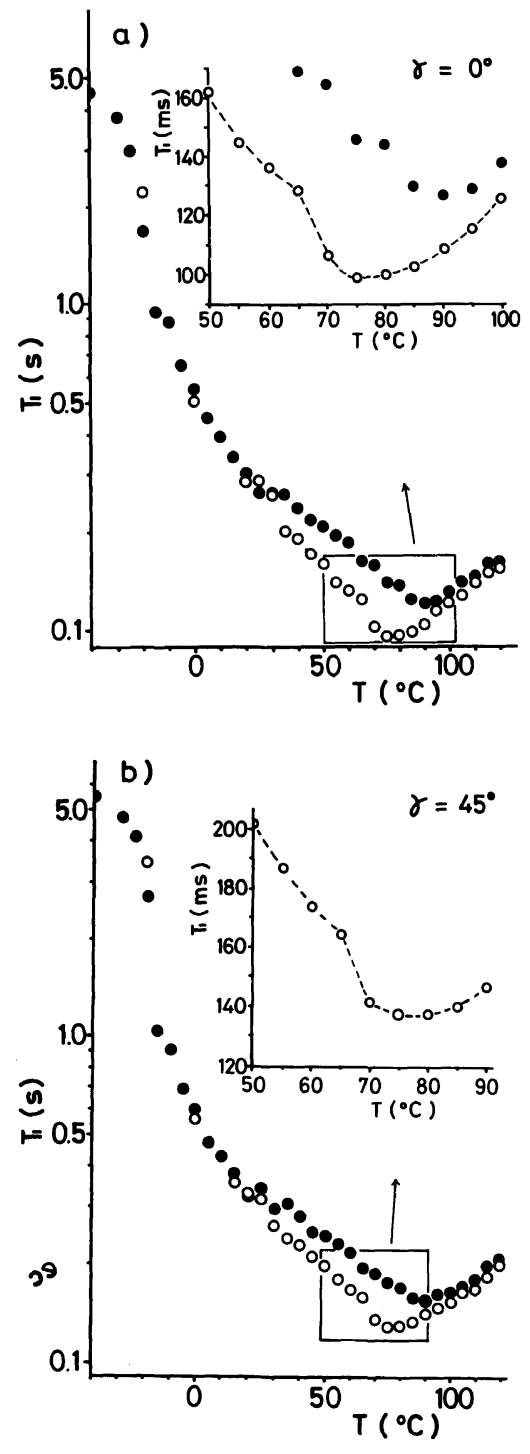

corresponding in the $\uparrow$-process. The value of the $T_{1}$ for each angle $\gamma$ decreases gradually from $120^{\circ} \mathrm{C}$ and is minimal at $75^{\circ} \mathrm{C}(\downarrow)$. After the $T_{1}$ increases abruptly between 70 and $65^{\circ} \mathrm{C}$ $(\downarrow)$, it increases slowly and at $30^{\circ} \mathrm{C}$ returns to that in the $\uparrow$-process. A discontinuity is observed in the $T_{1}$ vs. $T$ curve for each angle $\gamma$ between 65 and $70^{\circ} \mathrm{C}(\downarrow)$ more clearly than that of $\mathrm{P}\left(\mathrm{VDF}_{52} / \mathrm{TrFE}_{48}\right)$. Figure 4 shows the $T_{1}$ ploted against $T^{-1}$ in $\mathrm{P}\left(\mathrm{VDF}_{65} / \mathrm{TrFE}_{35}\right)$ for $\gamma=0,45$, and $90^{\circ}$ in the $\downarrow$-process. $T_{1}$ increases

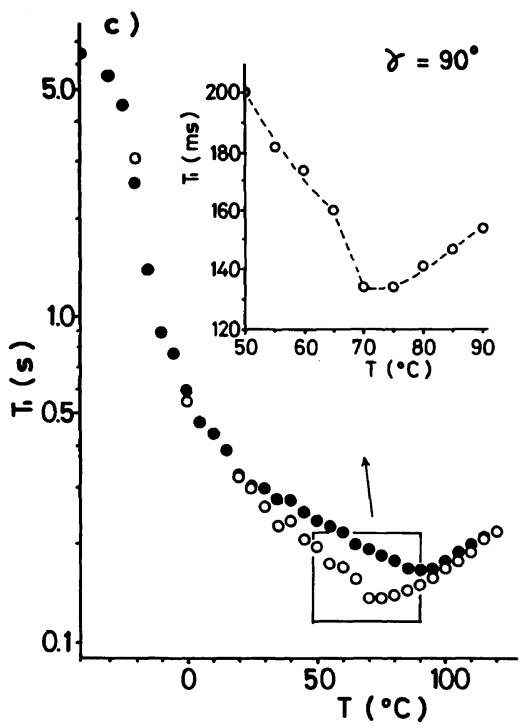

Figure 3. Temperature dependences of $T_{1}$ for $\mathrm{P}\left(\mathrm{VDF}_{65} / \mathrm{TrFE}_{35}\right)$ : (a) $\gamma=0^{\circ}$, (b) $\gamma=45^{\circ}$, and (c) $\gamma=90^{\circ}$. The filled and unfilled circles indicate $T_{1}$ in the $\uparrow$ - and $\downarrow$ processes. The insets show the behavior of $T_{1}$ near the transition temperature in the $\uparrow$-process $(\bigcirc)$ and in the $\downarrow$ process $\left(--\mathrm{O}^{-}-\right)$.

Table I. Classification of spin relaxation processes of $\mathrm{P}\left(\mathrm{VDF}_{52} / \mathrm{TrFE}_{48}\right)$

\begin{tabular}{|c|c|c|c|}
\hline \multicolumn{2}{|c|}{$\uparrow$-Process } & \multicolumn{2}{|c|}{$\downarrow$-Process } \\
\hline \multirow{2}{*}{$\begin{array}{c}\text { Relaxation } \\
\text { process }\end{array}$} & $\begin{array}{c}\text { Temperature } \\
\text { range }\end{array}$ & Relaxation & $\begin{array}{c}\text { Temperature } \\
\text { range }\end{array}$ \\
\hline & ${ }^{\circ} \mathrm{C}$ & & ${ }^{\circ} \mathrm{C}$ \\
\hline$\beta$ & $30-55^{a}$ & $\beta$ & $30-55^{a}$ \\
\hline$\alpha_{t}$ & $60-95$ & $\alpha_{t}$ & $60-95$ \\
\hline$\alpha_{b}^{\prime}$ & $95-120^{b}$ & $\alpha_{b}^{\prime}$ & $95-120^{b}$ \\
\hline
\end{tabular}

a Assumed that the temperature region of the $\beta$ relaxation is below $55^{\circ} \mathrm{C}$ where the value of $T_{1}$ varies discontinuously.

b Determined from the linearity of the $\ln T_{1}$ against reciprocal temperature in the paraelectric phase.

in order of the angle $\gamma=0,45$, and $90^{\circ}$ between 120 and $70^{\circ} \mathrm{C}(\downarrow)$, and in order of the angle $\gamma=0,90$, and $45^{\circ}$ below $65^{\circ} \mathrm{C}(\downarrow) . \mathrm{P}_{\left(\mathrm{VDF}_{65}\right.} /$ $\mathrm{TrFE}_{35}$ ) also exhibits three kinds of spinlattice relaxations in the $\downarrow$-process, that is, $\beta(\downarrow)$, $\alpha_{t}(\downarrow)$, and $\alpha_{b}^{\prime}(\downarrow)$, which take place in the three 


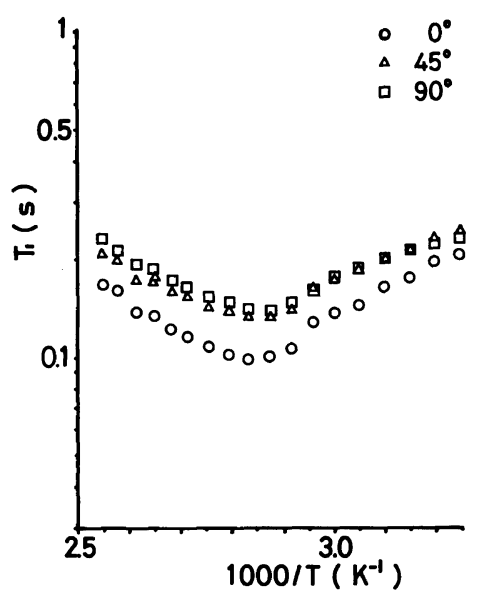

Figure 4. Plots of $T_{1}$ against reciprocal temperature for $\mathrm{P}\left(\mathrm{VDF}_{65} / \mathrm{TrFE}_{35}\right)$. The circles, triangles and squares indicate $T_{1}$ for $\gamma=0,45$, and $90^{\circ}$, respectively.

Table II. Classification of spin relaxation processes of $\mathrm{P}\left(\mathrm{VDF}_{65} / \mathrm{TrFE}_{35}\right)$

\begin{tabular}{cccc}
\hline & \multicolumn{2}{c}{$\downarrow$-Process } & \multicolumn{2}{c}{$\begin{array}{c}\text { Tempecess } \\
\begin{array}{c}\text { Relaxation } \\
\text { process }\end{array}\end{array}$} & $\begin{array}{c}\text { Temperature } \\
\text { range }\end{array}$ & $\begin{array}{c}\text { Relaxation } \\
\text { process }\end{array}$ & $\begin{array}{c}{ }^{\circ} \mathrm{C} \\
{ }^{\circ} \mathrm{C}\end{array}$ & & ${ }^{\circ}$ \\
\hline$\beta$ & $30-79^{\mathrm{a}}$ & $\beta$ & $30-65^{\mathrm{b}}$ \\
$\alpha_{\mathrm{t}}$ & $85-100$ & $\alpha_{\mathrm{t}}$ & $70-95$ \\
$\alpha_{\mathrm{b}}^{\prime}$ & $100-120^{\mathrm{c}}$ & $\alpha_{\mathrm{b}}^{\prime}$ & $95-120^{\mathrm{c}}$ \\
\hline
\end{tabular}

a Assumed that since no the $\beta(\uparrow)$ relaxation can be separated from the $\alpha_{t}(\uparrow)$ relaxation, the temperature region is below $79^{\circ} \mathrm{C}$ where a discontinuous line narrowing was observed in NMR line width measurement. $^{8}$

b Determined that the temperature region of the $\beta(\downarrow)$ is below $65^{\circ} \mathrm{C}$ where the value of $T_{1}$ increases discontinuously.

c Determined from the linearity of the $\ln T_{1}$ against reciprocal temperature in the paraelectric phase.

temperature ranges from 30 to $65^{\circ} \mathrm{C}(\downarrow), 70$ to $95^{\circ} \mathrm{C}(\downarrow)$, and 95 to $120^{\circ} \mathrm{C}(\downarrow)$, respectively. In Table II, the temperature ranges of the $\beta, \alpha_{t}$, and $\alpha_{b}^{\prime}$ relaxations in the $\uparrow$ - and $\downarrow$-processes are summarized.

Figures 5a, b, and c show the $\ln T_{1}$ vs. $\ln \omega$ curves in $\mathrm{P}\left(\mathrm{VDF}_{x} / \operatorname{TrFE}_{100-x}\right)$ with the VDF

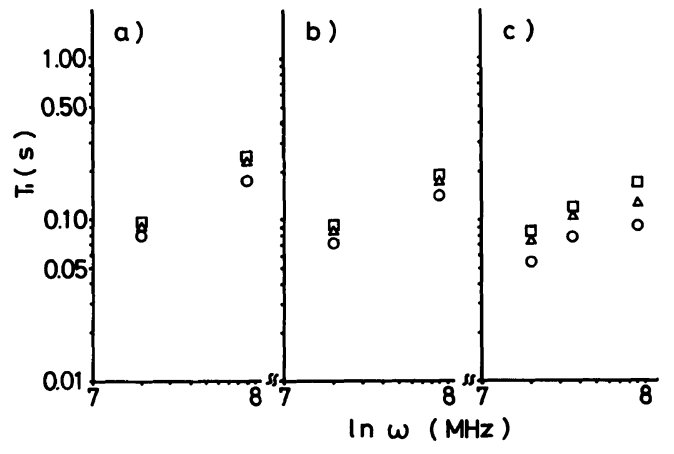

Figure 5. Plots of $\ln T_{1} v s . \ln \omega$ at $110^{\circ} \mathrm{C}(\downarrow)$ for three

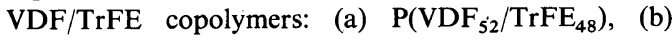
$\mathrm{P}\left(\mathrm{VDF}_{65} / \mathrm{TrFE}_{35}\right)$, and (c) $\mathrm{P}\left(\mathrm{VDF}_{72} / \mathrm{TrFE}_{28}\right)$. The circles, triangles and squares indicate $T_{1}$ for $\gamma=0,45$, and $90^{\circ}$, respectively.

contents of 52,65 , and $72 \mathrm{~mol} \%$ at $110^{\circ} \mathrm{C}(\downarrow)$. The $T_{1}$ vs. $\ln \omega$ curves of $\mathrm{P}\left(\mathrm{VDF}_{72} / \mathrm{TrFE}_{28}\right)$ have been given in Part I of this series. ${ }^{3}$ The circle, triangle and rectangle in each figure indicate the values of $T_{1}$ in the $\downarrow$-process for $\gamma=0,45$, and $90^{\circ}$, respectively. $T_{1}$ for each copolymer becomes longer with increasing of $\omega$ and $\gamma$.

\section{DISCUSSION}

\section{The Behavior of Spin-Lattice Relaxation Time $T_{1}$ near $T_{\mathrm{c}}$}

Thermal hysteresis of the $T_{1} v s$. $T$ curve becomes smaller in order of the VDF content from 72 to $52 \mathrm{~mol} \%$. In Table III, the temperatures of the $T_{1}$ minima, $T_{\mathrm{c}}(\uparrow)$ and $T_{\mathrm{c}}(\downarrow)$, in $\mathrm{P}\left(\mathrm{VDF}_{x} / \mathrm{TrFE}_{100-x}\right)$ with $x$ of 53,65 , and $72 \mathrm{~mol} \%$ are listed. The temperatures of the $T_{1}$ minima for $\mathrm{P}\left(\mathrm{VDF}_{72} / \mathrm{TrFE}_{28}\right)$ were determined in the previous paper. ${ }^{3}$ Both $T_{\mathrm{c}}(\uparrow)$ and $T_{\mathrm{c}}(\downarrow)$ shift to lower temperature as the VDF content decreases. They agree approximately with the corresponding transition temperatures obtained from the dielectric ${ }^{6}$ and $\mathrm{DSC}^{6}$, and NMR line width ${ }^{7,8}$ measurements. Therefore, the $T_{1}$ for the $\alpha_{\mathrm{t}}$-relaxation in each copolymer is dully minimul at $T_{\mathrm{c}}$ due to the ferroelectric phase transition. This dull minimum of the $T_{1}$ suggests that the critical slow- 
Table III. The temperatures of $T_{1}$ minima for three VDF/TrFE copolymers with the VDF contents of 52,65 , and $72 \mathrm{~mol} \%$ in the heating and cooling processes

\begin{tabular}{|c|c|c|c|c|c|c|}
\hline & \multicolumn{2}{|c|}{$52 / 48$} & \multicolumn{2}{|c|}{$65 / 35$} & \multicolumn{2}{|c|}{$72 / 28$} \\
\hline & $T_{\mathrm{c}}(\uparrow)$ & $T_{\mathrm{c}}(\downarrow)$ & $T_{\mathrm{c}}(\uparrow)$ & $T_{\mathrm{c}}(\downarrow)$ & $T_{\mathrm{c}}(\uparrow)$ & $T_{\mathrm{c}}(\downarrow)$ \\
\hline & ${ }^{\circ} \mathrm{C}$ & ${ }^{\circ} \mathrm{C}$ & ${ }^{\circ} \mathrm{C}$ & ${ }^{\circ} \mathrm{C}$ & ${ }^{\circ} \mathrm{C}$ & ${ }^{\circ} \mathrm{C}$ \\
\hline$T_{1}$ & 68 & 68 & 90 & 75 & 115 & 79 \\
\hline$\Delta H^{\mathrm{a}}$ & 63 & 63 & 89 & 70 & 116 & 75 \\
\hline $\mathrm{DSC}^{\mathrm{b}}$ & 60 & 62 & 70 & 64 & 114 & 75 \\
\hline $1 / \Delta \varepsilon^{\mathrm{b}}$ & 79 & 73 & 102 & 69 & 134 & 84 \\
\hline
\end{tabular}

a Observed from the discontinuous narrowing of the NMR line width. ${ }^{7,8}$

b Observed from the minimum of the reciprocal dielectric strength $1 / \Delta \varepsilon^{6}$ and the endotherm starting point on the DSC curve. ${ }^{6}$

ing down of the molecular motion due to polarization fluctuation near $T_{\mathrm{c}}$ is affected by a first order of the phase transition. The value of $T_{1}$ in the phase transition region becomes larger in order of VDF content $x=72,52$, and $65 \mathrm{~mol} \%$. While, the dielectric relaxation strength $\Delta \varepsilon$ near $T_{\mathrm{c}}$ obtained from the dielectric measurements ${ }^{6,9}$ increases in order of VDF content $x=72,52$, and $65 \mathrm{~mol} \%$. It was previously ${ }^{10}$ estimated from the values of $T_{1}$ and $\Delta \varepsilon$ at $T_{\mathrm{c}}$ for each copolymer that the correlation energy in the spatial variation of polarization due to the critical fluctuation becomes larger in order of VDF content $x=$ 72,65 , and $52 \mathrm{~mol} \%$. This means that the spatial inhomogeneity due to the polarization fluctuation is reflected in $T_{1}$ near $T_{\mathrm{c}}$.

The $\beta$-relaxations for $\mathrm{P}\left(\mathrm{VDF}_{52} / \mathrm{TrFE}_{48}\right)$ and $\mathrm{P}\left(\mathrm{VDF}_{65} / \mathrm{TrFE}_{35}\right)$ appear below $T_{\mathrm{c}}$ in the $\uparrow-$ and $\downarrow$-processes, respectively. The $T_{1} v s$. $T$ curve in each copolymer does not take the minimum value in the $\beta$ relaxation region. While the minimization of $T_{1}$ for the relaxation was observed at $88^{\circ} \mathrm{C}$ in the heating process for $\mathrm{P}\left(\mathrm{VDF}_{72} / \mathrm{TrFE}_{28}\right)$ only. ${ }^{3}$ This is attributed to the finding that the temperature of $88^{\circ} \mathrm{C}$ for the $\beta$-relaxation is higher than all $T_{\mathrm{c}}$ 's, except the $T_{\mathrm{c}}(\uparrow)$ in $\mathrm{P}\left(\mathrm{VDF}_{72} / \mathrm{TrFE}_{28}\right)$ as shown in
Table II. The values of $T_{1}$ for the $\beta$ relaxation become larger in order. of the VDF content $x=72,52$, and $65 \mathrm{~mol}^{\circ} \%$. The $\beta$ relaxation may be due to the molecular motion of the chain backbone in partially crystalline regions. ${ }^{6}$ The 1-D diffusion motion of conformational defects in the paraelectric phase.

In the previous papers, ${ }^{3,7}$ the formular for $T_{1}$ of an isolated spin pair modulated by a onedimensional (1-D) diffusion motion of conformational defects was given, at the high temperature limit, as follows:

$$
\begin{aligned}
\ln T_{1}= & -1 / 2 \ln \tau_{\mathrm{D}}+1 / 2 \ln \omega \\
& -\ln \left\{(1+2 \sqrt{2})+6 \cos ^{2} \gamma\right. \\
& \left.+(1-2 \sqrt{2}) \cos ^{4} \gamma\right\}-\ln A
\end{aligned}
$$

where $A=9 / 128 \gamma^{4} \hbar^{2} I(I+1) r^{-6}$ and $\omega=2 \pi f_{\mathrm{r}}$. The $\tau_{\mathbf{D}}$ is defined as

$$
\tau_{\mathrm{D}}=l_{\mathrm{d}}^{2} / D
$$

where $l_{\mathrm{d}}$ is a mean distance between a given isolated spin pair and the nearest conformational defect and $D$ is the diffusion coefficient. Inserting eq 2 into eq $1, T_{1}$ is represented by

$$
\begin{aligned}
\frac{1}{T_{1}} & =B \tau_{\mathrm{D}}^{1 / 2} \\
& =B \frac{l_{d}}{\sqrt{D}}
\end{aligned}
$$

where

$$
\begin{aligned}
B= & A^{-1} \omega^{-1 / 2}\{(1+2 \sqrt{2}) \\
& \left.+6 \cos ^{2} \gamma+(1+2 \sqrt{2}) \cos ^{4} \gamma\right\}
\end{aligned}
$$

Assuming that $\tau_{\mathrm{D}}$ is expressed by Arrhenius type, $\tau_{\mathrm{D}}=\tau_{0} \exp (\Delta E / R T)$, eq 1 becomes

$$
\ln T_{1}=\frac{1}{2}\left(\frac{\Delta E}{R}\right) \frac{1}{T}-\ln B
$$

where $\Delta E$ is an activation energy of the 1-D diffusion motion.

If each $\ln T_{1}$ vs. $\ln \omega$ curve in Figure 5 is linear, the slope of each line is determined from the experimental data by using the least square method. Table IV illustrates the 
Table IV. The averaged slopes, ' $\bar{a}$ 's, ' of $\ln T_{1} v s$. $\ln \omega$ at $110^{\circ} \mathrm{C}(\downarrow)$ for the VDF/TrFE copolymers with the VDF contents of 52,65 , and $72 \mathrm{~mol} \%$, and the averaged activation energy of the $\alpha_{b}^{\prime}$ relaxation in each copolymer in the cooling process

\begin{tabular}{clll}
\hline $\mathrm{VDF} / \mathrm{mol} \%$ & 52 & 65 & $72^{\mathrm{a}}$ \\
\hline $\bar{a}$ & 0.58 & 0.49 & 0.50 \\
$\Delta E / \mathrm{kcal} \mathrm{mol}^{-1}$ & 8.7 & 8.7 & 8.2 \\
\hline
\end{tabular}

a The values of $\bar{a}$ and $\Delta E$ for $\mathrm{P}\left(\mathrm{VDF}_{72} / \mathrm{TrFE}_{28}\right)$ were determined in Part I of this series. ${ }^{3}$

average slopes, $\bar{a}$ 's for the copolymers with VDF contents of 52,65 , and $72 \mathrm{~mol} \%$. The $\bar{a}$ values for $\mathrm{P}\left(\mathrm{VDF}_{52} / \mathrm{TrFE}_{48}\right)$ and $\mathrm{P}\left(\mathrm{VDF}_{65} /\right.$ $\mathrm{TrFE}_{35}$ ) are respectively 0.58 and 0.49 , which are approximately equal the theoretical value 0.5 obtained from eq 1 .

The plots of the $\ln T_{1}$ against $T^{-1}$ for $\gamma=0$, 45 , and $90^{\circ}$ in each copolymer yield a straight line in the temperature range from 95 to $120^{\circ} \mathrm{C}$ $(\downarrow)$ as shown in Figures 2 and 4 . The values of $T_{1}$ become longer in order of the $\gamma=0,45$, and $90^{\circ}$. The averaged ratios of $T_{1}$ 's for $\gamma=45$ and $90^{\circ}$ to that for $\gamma=0^{\circ}$ in the temperature range are respectively 1.26 and 1.38 for $\mathrm{P}\left(\mathrm{VDF}_{52}\right)$ $\left.\mathrm{TrFE}_{48}\right)$, and 1.27 and 1.42 for $\mathrm{P}\left(\mathrm{VDF}_{65} /\right.$ $\mathrm{TrFE}_{35}$ ). Comparing these ratios with the ratios of 1.28 and 1.41 for $\mathrm{P}\left(\mathrm{VDF}_{72} / \mathrm{TrFE}_{28}\right)$, we can see that these ratios of $T_{1}$ 's in the copolymers are almost independent of the VDF content. The experimental values of these ratios agree qualitatively with the theoretical ratios of 1.26 and 2.1 , which is calculated from eq 1.

Using eq 4 and the values of the slope of $\ln T_{1}$ vs. $T^{-1}$ in each copolymer obtained for $\gamma=0,45$, and $90^{\circ}$, the activation energy of the 1-D diffusion motion is calculated. The averaged activation energies for $\mathrm{P}\left(\mathrm{VDF}_{52} /\right.$ $\left.\mathrm{TrFE}_{48}\right)$ and $\mathrm{P}\left(\mathrm{VDF}_{65} / \mathrm{TrFE}_{35}\right)$ are listed in Table IV together with that in $\mathrm{P}\left(\mathrm{VDF}_{72} /\right.$ $\mathrm{TrFE}_{28}$ ). The activation energy of about 8.5 $\mathrm{kcal} / \mathrm{mol}$ is almost independent of the VDF content. Therefore, the existence of a 1-D

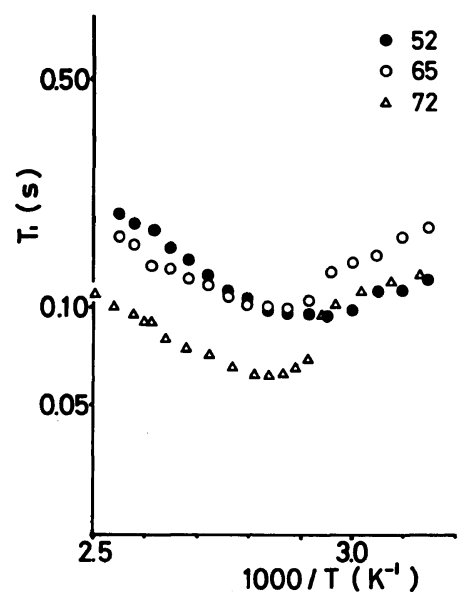

Figure 6. The effect of the VDF content on the $T_{1}$ behavior of the VDF/TrFE copolymer in the cooling process. $\mathrm{P}\left(\mathrm{VDF}_{52} / \mathrm{TrFE}_{48}\right), \quad \mathrm{P}\left(\mathrm{VDF}_{65} / \mathrm{TrFE}_{35}\right)$, and $\mathrm{P}\left(\mathrm{VDF}_{72} / \mathrm{TrFE}_{28}\right)$.

diffusion motion of the conformational defects in the paraelectric phase is confirmed from the reasonable agreement between the theoretical and the experimental relations of $T_{1}$ to $\omega, \gamma$ and $T^{-1}$.

\section{Influence of the VDF Content on a One- Dimensional Diffusion Motion of the Con- formational Defect}

Figure 6 shows the $\ln T_{1}$ plotted against $T^{-1}$ in $\mathrm{P}\left(\mathrm{VDF}_{x} / \mathrm{TrFE}_{100-x}\right)$ with $x$ of 52,65 , and $72 \mathrm{~mol} \%$ for $\gamma=0^{\circ}$. In the high temperature region in the paraelectric phase, the $T_{1}$ becomes longer as the VDF content decreases. $T_{1}$ 's for $\gamma=45$ and $90^{\circ}$ also depend on the VDF content in a similar way to that for $\gamma=0^{\circ}$. Let us express the observed $\left(1 / T_{1}\right)_{\mathrm{ob}}$ at $110^{\circ} \mathrm{C}(\downarrow)$ and the VDF content ' $x$ ' in P$\left(\mathrm{VDF}_{x} / \operatorname{TrFE}_{100-x}\right)$ as values relative to corresponding those in $\mathrm{P}\left(\mathrm{VDF}_{72} / \mathrm{TrFE}_{28}\right)$, which are referred to as $\left(1 / T_{1}\right)_{72}$ and 72 , respectively. Then, two parameters, $P_{\mathrm{ob}}(\gamma)$ and $\xi$ are defined by the following equations

$$
P_{\mathrm{ob}}(\gamma)=\frac{\left(\frac{1}{T_{1}}\right)_{\mathrm{ob}}(\gamma)}{\left(\frac{1}{T_{1}}\right)_{72}(\gamma)}
$$


Table V. Scaling factors $\bar{P}_{\text {ob }}$ and $\xi^{2}$ of $\left(1 / T_{1}\right)$ 's for the VDF/TrFE copolymers with the VDF contents of 52,65 and $72 \mathrm{~mol} \%$ to that for $\mathrm{P}\left(\mathrm{VDF}_{72} / \mathrm{TrFE}_{28}\right)$ at $110^{\circ} \mathrm{C}$ in the cooling process

\begin{tabular}{cccc}
\hline $\mathrm{VDF} / \mathrm{mol} \%$ & 52 & 65 & 72 \\
\hline$P_{\mathrm{ob}}^{\mathrm{a}}$ & 0.52 & 0.65 & 1 \\
$\xi^{2 \mathrm{~b}}$ & 0.52 & 0.81 & 1 \\
\hline
\end{tabular}

a $\bar{P}_{\mathrm{ob}}=(1 / 3) \sum_{\gamma} P_{\mathrm{ob}}(\gamma), \gamma=0,45$ and $90^{\circ}$.

b $\xi=x / 72$, in which $x$ is the VDF molar content.

$$
\xi=x / 72
$$

The $P_{\mathrm{ob}}(\gamma)$ 's calculated from the experimental data of the copolymers do not almost depend on the angle $\gamma$ in the temperature region between 100 and $120^{\circ} \mathrm{C}(\downarrow)$. The values of the averaged $\bar{P}_{\mathrm{ob}}, \quad \bar{P}_{\mathrm{ob}}=1 / 3 \sum_{\gamma} P_{\mathrm{ob}}(\gamma) \quad(\gamma=0,45$, and $90^{\circ}$ ), and $\xi^{2}$ against the VDF content of ' $x$ ' are listed in Table V. The values of $\bar{P}_{\mathrm{ob}}$ coincide approximately with those of $\xi^{2}$. Therefore, using the parameter $\xi^{2}$ in the stead of the $\bar{P}_{\text {ob }}$, the $\left(1 / T_{1}\right)_{72}$ is written by

$$
\left(\frac{1}{T_{1}}\right)_{72}(\gamma)=\xi^{-2}\left(\frac{1}{T_{1}}\right)_{\mathrm{ob}}(\gamma)
$$

Therefore, using eq 1 and 6 , the correlation time $\tau_{D}$ for each copolymer transforms according to

$$
\tau_{\mathrm{D} 72}=\xi^{-4} \tau_{\mathrm{D}}
$$

where $\tau_{\mathrm{D} 72}$ is the correlation time for $\mathbf{P}$ $\left(\mathrm{VDF}_{72} / \mathrm{TrFE}_{28}\right)$. Therefore, the VDF content is strongly related to the $T_{1}$ and $\tau_{\mathrm{D}}$. The values of $T_{1}$ at $110^{\circ} \mathrm{C}(\downarrow)$ and $120^{\circ} \mathrm{C}(\downarrow)$ for $\mathrm{P}\left(\mathrm{VDF}_{52} /\right.$ $\left.\mathrm{TrFE}_{48}\right)$ and $\mathrm{P}\left(\mathrm{VDF}_{65} / \mathrm{TrFE}_{35}\right)^{\circ}$ which are calculated from that in $\mathrm{P}\left(\mathrm{VDF}_{72} / \mathrm{TrFE}_{28}\right)$ by using the scaling factor are shown in the parentheses in Table VI and are compared with the values of $\left(T_{1}\right)_{\mathrm{ob}}$ for $\mathrm{P}\left(\mathrm{VDF}_{x}\right)$ $\left.\operatorname{TrFE}_{100-x}\right)\left(x=52\right.$ and $\left.65 \mathrm{~mol}^{\circ} \%\right)$ observed at 110 and $120^{\circ} \mathrm{C}$ in the cooling process. The agreements between the calculated values and experimental data are qualitatively good although the calculated values for
Table VI. Comparison between the theoretical and the experimental values of $T_{1}$ 's in the VDF/TrFE copolymers for $\gamma=0,45$, and $90^{\circ}$ at 110 and $120^{\circ} \mathrm{C}$ in the cooling process. The values of $T_{1}$ which are shown in the parentheses are calculated from the experimental value

\begin{tabular}{|c|c|c|c|c|}
\hline \multirow[b]{2}{*}{$T$} & \multirow[b]{3}{*}{$\left(^{\circ}\right)$} & $72 / 28$ & $65 / 35$ & $52 / 48$ \\
\hline & & $T_{1}$ & $T_{1}$ & $T_{1}$ \\
\hline${ }^{\circ} \mathrm{C}$ & & $\mathrm{ms}$ & $\mathrm{ms}$ & $\mathrm{ms}$ \\
\hline \multirow{5}{*}{110} & 0 & 91.8 & $\begin{array}{c}134.2 \\
(113.0)\end{array}$ & $\begin{array}{r}178.0 \\
(176.5)\end{array}$ \\
\hline & \multirow[t]{2}{*}{45} & 120.9 & 169.4 & 225.0 \\
\hline & & & (149.3) & $(231.0)$ \\
\hline & \multirow[t]{2}{*}{90} & 127.0 & 194.7 & 248.0 \\
\hline & & & $(156.8)$ & $(244.2)$ \\
\hline \multirow{6}{*}{120} & \multirow[t]{2}{*}{0} & 101.0 & 164.7 & 194.8 \\
\hline & & & (124.7) & (194.2) \\
\hline & \multirow[t]{2}{*}{45} & 129.8 & 209.3 & 251.2 \\
\hline & & & $(160.2)$ & (249.6) \\
\hline & \multirow[t]{2}{*}{90} & 147.0 & 223.4 & 288.5 \\
\hline & & & $(185.1)$ & $(282.7)$ \\
\hline
\end{tabular}
in $\mathrm{P}\left(\mathrm{VDF}_{72} / \mathrm{TrFE}_{28}\right)$ by using the scaling factor $\xi$ (see eq 6)

$\mathrm{P}\left(\mathrm{VDF}_{65} / \mathrm{TrFE}_{35}\right)$ are smaller than the experimental data.

\section{CONCLUSION}

For the drawn films of $\mathrm{P}\left(\mathrm{VDF}_{52} / \mathrm{TrFE}_{48}\right)$ and $\mathrm{P}\left(\mathrm{VDF}_{65} / \mathrm{TrFE}_{35}\right)$, three kinds of the spin lattice relaxations were observed below the Curie temperature $T_{\mathrm{c}}$, around $T_{\mathrm{c}}$ and in a higher temperature region above $T_{\mathrm{c}}$, respectively, which are referred to as $\beta, \alpha_{t}$, and $\alpha_{b}^{\prime}$. They were compared with those of $\mathrm{P}\left(\mathrm{VDF}_{72}\right)$ $\mathrm{TrFE}_{28}$ ) reported in Part $\mathrm{I}$ of this series. ${ }^{3}$ The values of $T_{1}$ for the $\beta$ relaxation became larger in order of the VDF content $x=72,52$, and $65 \mathrm{~mol} \%$, which may be attributed to the motion of the chain backbone in partially crystalline regions. The $T_{1}$ for $\alpha_{\mathrm{t}}$ relaxation in each copolymer was minimal at $T_{\mathrm{c}}$ due to the phase transition. The $T_{1}$ vs. $T$ curve showed more largely thermal hysteresis with increasing of VDF content, of which the temperature of 
the $T_{1}$ minimum agreed approximately with the Curie temperature. The value of $T_{1}$ in the phase transition region became smaller in order of 65,52 , and $72 \mathrm{~mol} \%$.

The $T_{1}$ 's for the $\alpha_{\mathrm{b}}^{\prime}$ relaxations in $\mathrm{P}\left(\mathrm{VDF}_{52} /\right.$ $\left.\mathrm{TrFE}_{48}\right)$ and $\mathrm{P}\left(\mathrm{VDF}_{65} / \mathrm{TrFE}_{35}\right)$ were analyzed, respectively, on the basis of Bloembergen's thoery, ${ }^{4}$ in terms of the non-exponential correlation function obtained by Hunt and Powles. ${ }^{5}$ The existence of the one-dimensional diffusion motion of the conformational defect in each copolymer was verified from the reasonable agreements between the theoretical and experimental results. The activation energy of the 1-D diffusion motion did not depend on the VDF content, which was about $8.5 \mathrm{kcal} \mathrm{mol}^{-1}$. The $\left(1 / T_{1}\right)$ 's and the correlation times of the local field modulated by the 1-D diffusion motion in $\mathrm{P}\left(\mathrm{VDF}_{52} / \mathrm{TrFE}_{48}\right)$ and $\mathrm{P}\left(\mathrm{VDF}_{65} / \mathrm{TrFE}_{35}\right)$ were converted approximately to those in $\mathrm{P}\left(\mathrm{VDF}_{72} / \mathrm{TrFE}_{28}\right)$ with the scaling factors of $\xi^{-2}$ and $\xi^{-4}$, respectively, in which $\xi$ is the ratios of the VDF content of 52 and $65 \mathrm{~mol} \%$ to $72 \mathrm{~mol} \%$. This means that the VDF content has a great influence on the 1-D diffusion motion.

Acknowledgement. The authors are grateful to Drs. Jun Koizumi, Junichi Sako, Toshiharu Yagi and Yoshihide Higashihata of the Daikin Kogyo Co., Ltd., for supplying samples of $\mathrm{VDF} / \mathrm{TrFE}$ copolymers.

\section{REFERENCES}

1. K. Tashiro, T. Takano, M. Kobayashi, Y. Chatani, and H. Tadokoro, Ferroelectrics, 57, 297 (1984).

2. A. Odajima, Ferroelectrics, 57, 159 (1984).

3. F. Ishii and A. Odajima, Polym. J., 18, 539 (1986).

4. N. Bloenbergen, Phys. Rev., 104, 1542 (1956).

5. B. I. Hunt and J. G. Powles, Proc. Phys. Soc., 88, 513 (1971).

6. N. Koizumi, N. Haikawa, and H. Habuta, Ferroelectrics, 57, 99 (1984).

7. F. Ishii, A. Odajima, and H. Ohigashi, Polym. J., 15, 875 (1983).

8. F. Ishii and A. Odajima, Polym. Prepr., Jpn., 34, 935 (1985).

9. T. Furukawa, M. Ohuchi, A. Chiba, and M. Date, Macromolecules, 17, 1384 (1984).

10. F. Ishii and A. Odajima, Rep. Prog. Polym. Phys., Jpn., 27, 537 (1984). 